\title{
Creep measurements confirm steady flow after stress maximum in extension of branched polymer melts
}

Javier Alvarez, Nicolas; Román Marín, José Manuel; Huang, Qian; Michelsen, Michael Locht; Hassager, Ole

Published in:

Physical Review Letters

Link to article, DOI:

10.1103/PhysRevLett.110.168301

Publication date:

2013

Document Version

Publisher's PDF, also known as Version of record

Link back to DTU Orbit

Citation (APA):

Javier Alvarez, N., Román Marín, J. M., Huang, Q., Michelsen, M. L., \& Hassager, O. (2013). Creep measurements confirm steady flow after stress maximum in extension of branched polymer melts. Physical Review Letters, 110(16), [168301]. https://doi.org/10.1103/PhysRevLett.110.168301

\section{General rights}

Copyright and moral rights for the publications made accessible in the public portal are retained by the authors and/or other copyright owners and it is a condition of accessing publications that users recognise and abide by the legal requirements associated with these rights.

- Users may download and print one copy of any publication from the public portal for the purpose of private study or research.

- You may not further distribute the material or use it for any profit-making activity or commercial gain

- You may freely distribute the URL identifying the publication in the public portal 


\title{
Creep Measurements Confirm Steady Flow after Stress Maximum in Extension of Branched Polymer Melts
}

\author{
Nicolas J. Alvarez, José Manuel Román Marín, Qian Huang, Michael Locht Michelsen, and Ole Hassager* \\ Department of Chemical and Biochemical Engineering, Danish Polymer Center, \\ Technical University of Denmark, 2800 Kongens Lyngby, Denmark
}

(Received 8 November 2012; published 15 April 2013)

\begin{abstract}
We provide conclusive evidence of nonmonotonic mechanical behavior in the extension of long-chain branched polymer melts. While nonmonotonic behavior is known to occur for solids, for the case of polymeric melts, this phenomenon is in direct contrast with current theoretical models. We rule out the possibility of the overshoot being an experimental artifact by confirming the existence of steady flow after a maximum in the ratio of stress to strain rate versus strain under both constant stress and constant strainrate kinematics. This observation indicates the omission of important physics from current models for these industrially important materials, whose processing properties depend on extreme molecular extension.
\end{abstract}

DOI: 10.1103/PhysRevLett.110.168301

Introduction.-Predicting the viscoelastic properties of branched polymer melts from their molecular architecture remains one of the great challenges in polymer physics [1-3]. One outstanding problem is the lack of consensus of the evolution of stress in the start-up of constant strain-rate extensional flow. While one laboratory [4] has reported a maximum in stress followed by a steady value, other laboratories have legitimately questioned the maximum [5-7] and even challenged the existence of steady extensional flow [8,9]. The molecular origin of a stress maximum is unclear inasmuch as nonlinear models [10] specifically designed for branched polymers show a monotonic increase of stress in constant strain-rate extension.

Here, we report observations of extensional flow not with a constant strain rate but with a constant stress (creep). In addition to probing the transient behavior, the creep protocol also allows the measurement of the ultimate steady extensional viscosity. The uniqueness of the steady extensional viscosity independent of the start-up protocol is apparently not a settled matter as evidenced by the reporting of two viscosities at the same steady strain rate $[11,12]$. The experiments are compared to the small strain predictions $[13,14]$ to illustrate the departure from linear material behavior.

A major challenge in extensional rheometry is that large deformations are needed to approach a steady flow state. Deformations are measured in units of Hencky strain defined as $\epsilon=\ln \lambda$, where $\lambda=L / L_{0}$ is the stretch ratio, $L_{0}$ is the initial length, and $L$ is the final length of a given cylindrical shape. The strain rate $\dot{\epsilon}$ is the time derivative of the Hencky strain. In the classical controlled deformation extensional experiment, a constant $\dot{\epsilon}$ is imposed and the stress is monitored as a function of time. Typically, Hencky strains larger than four are required to establish a steady tensile stress. However, in many extensional flow devices, sample inhomogeneity will prevent control of the kinematics
PACS numbers: 47.57.Qk, 83.50.Jf, 83.60.Bc, 83.60.Df

at approximately the same Hencky strain, such that a steady flow cannot be observed.

Filament stretching rheometry.-The only extensional rheometer that can achieve absolute in situ control of the kinematics is the filament stretching rheometer (FSR) $[15,16]$. Traditionally, a FSR is operated in controlled deformation mode. In this work we have adapted the FSR to operate in controlled stress (creep) mode. While extensional creep testing has been performed with other devices $[11,12,17,18]$, this represents the first adaptation of a FSR device to operate in controlled stress mode. We use a low density polyethylene (LDPE, Lupolen 3020D) melt as model material. LDPE is a highly branched polymer with irregular long-chain branching $[19,20]$. An example of a stretched filament is shown in Fig. 1. Examples of imposed stress as a function of time are shown in Fig. 2.

Small deformations.-In the limit of small strain all nonlinear constitutive equations reduce to the framework of linear viscoelasticity (LVE). A frequently used LVE model is the multimode Maxwell model [21] whereby the shear stress $\sigma$ is a superposition of individual modes so that $\sigma=\sum \sigma_{i}$. The individual modes are given by

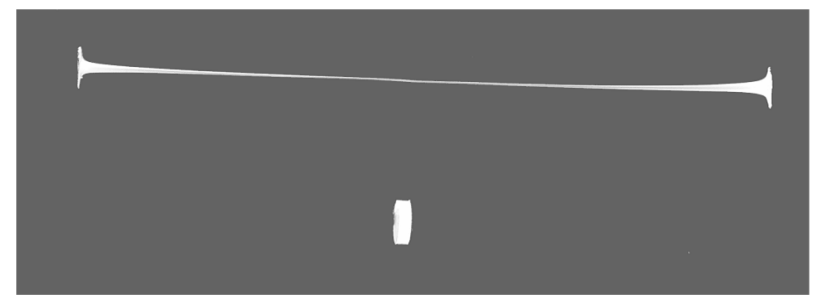

FIG. 1. Polyethylene sample before (bottom, diameter $9 \mathrm{~mm}$ ) and after (top) stretching. The midplane deformation corresponds to a Hencky strain of 6.7. The Hencky strain in the filament midplane is measured on-line by a laser micrometer during the experiment. 


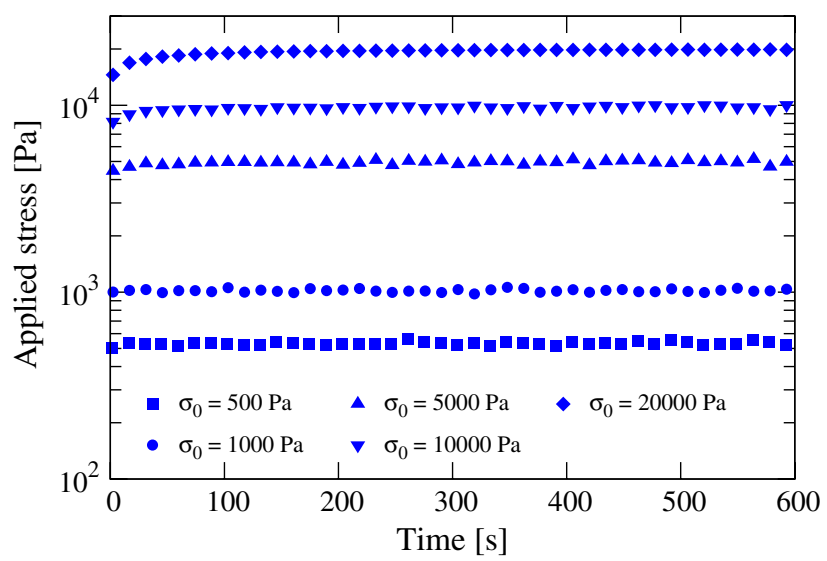

FIG. 2 (color online). Stress in the filament midplane measured as a function of time for five predefined stress levels.

$$
\sigma_{i}+\tau_{i} \frac{d \sigma_{i}}{d t}=g_{i} \tau_{i} \dot{\gamma}, \quad i=1,2, \ldots, n,
$$

where $\dot{\gamma}(t)$ is the shear rate. The moduli $g_{i}$ and relaxation times $\tau_{i}$ form a set of $2 n$ material parameters that completely characterize the LVE properties. Numerical values for the model melt [22] are reproduced in Table I.

In creep a total stress $\sigma_{0}$ is imposed at time $t=0$ such that Eq. (1) is augmented by the constraint,

$$
\sum_{i=1}^{n} \sigma_{i}(t)=\sigma_{0}
$$

The total shear deformation $\gamma(t)=\int_{0}^{t} \dot{\gamma}\left(t^{\prime}\right) d t^{\prime}$ is monitored as a function of time. The creep compliance $J(t)=$ $\gamma(t) / \sigma_{0}$ is then given by

$$
J(t)=J_{0}+\frac{t}{\eta_{0}}+\sum_{k=1}^{n-1} j_{k}\left[1-\exp \left(-t / \lambda_{k}\right)\right] .
$$

The parameters in the compliance expression are the instantaneous compliance $J_{0}=1 / \sum g_{i}$, the zero shearrate viscosity $\eta_{0}=\sum g_{i} \tau_{i}$, the $(n-1)$ retardation times $\lambda_{k}$, and the $(n-1)$ compliance coefficients $j_{k}$. The $\left(\lambda_{k}, j_{k}\right)$ are determined from Eqs. (A3) and (A4) derived in the Appendix. Numerical values are given in Table I. The retardation times are located in the intervals between the $n$ relaxation times so that $\lambda_{i} \in\left[\tau_{i} ; \tau_{i+1}\right]$,

TABLE I. Parameters for LDPE melt $\left(130^{\circ} \mathrm{C}\right)$.

\begin{tabular}{ccccc}
\hline \hline$i$ & $g_{i}(\mathrm{~Pa})$ & $\tau_{i}(\mathrm{~s})$ & $j_{i}\left(\mathrm{~Pa}^{-1}\right)$ & $\lambda_{i}(\mathrm{~s})$ \\
\hline 1 & $2.245 \times 10^{5}$ & $3.954 \times 10^{-3}$ & $2.310 \times 10^{-6}$ & $8.292 \times 10^{-3}$ \\
2 & $7.120 \times 10^{4}$ & $2.965 \times 10^{-2}$ & $2.932 \times 10^{-6}$ & $4.888 \times 10^{-2}$ \\
3 & $4.515 \times 10^{4}$ & $1.393 \times 10^{-1}$ & $5.215 \times 10^{-6}$ & $2.405 \times 10^{-1}$ \\
4 & $2.789 \times 10^{4}$ & $6.377 \times 10^{-1}$ & $9.893 \times 10^{-6}$ & $1.178 \times 10^{0}$ \\
5 & $1.628 \times 10^{4}$ & $2.893 \times 10^{0}$ & $2.059 \times 10^{-5}$ & $5.930 \times 10^{0}$ \\
6 & $8.451 \times 10^{3}$ & $1.322 \times 10^{1}$ & $5.180 \times 10^{-5}$ & $3.222 \times 10^{1}$ \\
7 & $4.039 \times 10^{3}$ & $6.880 \times 10^{2}$ & $4.599 \times 10^{-4}$ & $4.331 \times 10^{2}$ \\
8 & $7.132 \times 10^{2}$ & $1.066 \times 10^{3}$ & $\cdots$ & $\cdots$ \\
\hline \hline
\end{tabular}
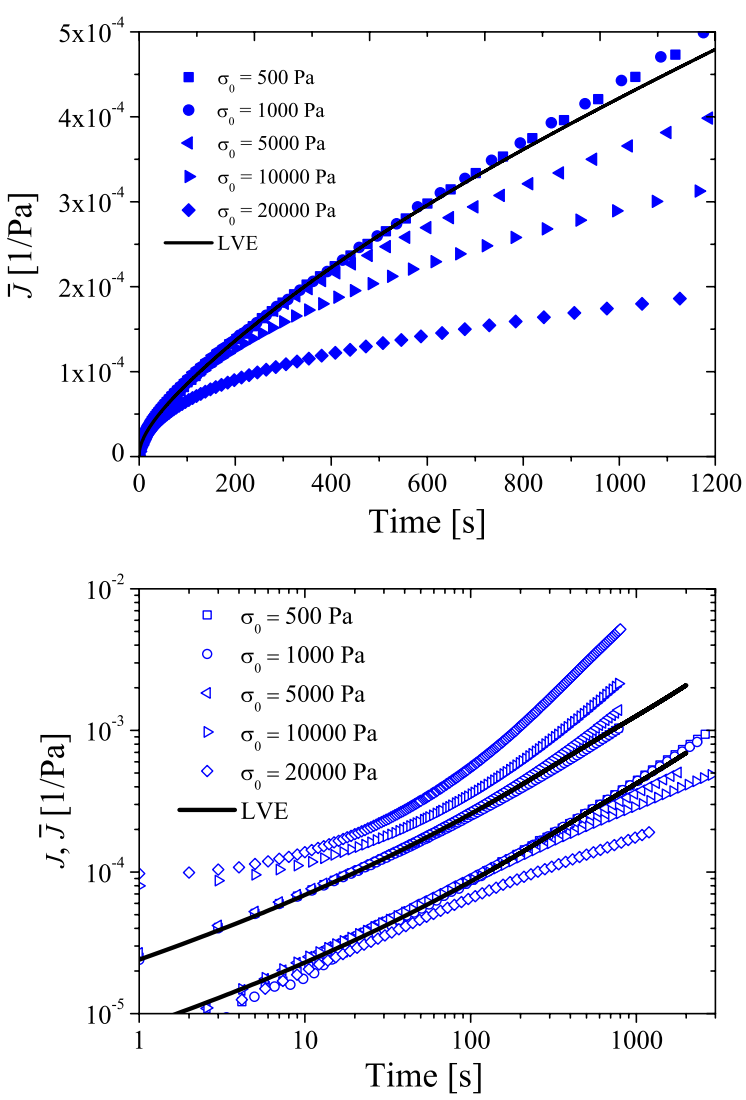

FIG. 3 (color online). Top: Extensional compliance $\bar{J}$ versus time at five applied stresses. Bottom: Comparison of shear compliance (top curves) and extensional compliance versus time for five applied stresses (log-log scales). The solid lines in both graphs correspond to the predicted LVE compliance from Eq. (3).

$i=1, \ldots, n-1$. In particular, the longest retardation time $\left(\lambda_{7}\right)$ is less than half the value of the longest relaxation time $\left(\tau_{8}\right)$ such that the transition to steady flow is faster in creep than in prescribed deformation. Hence, if the interest

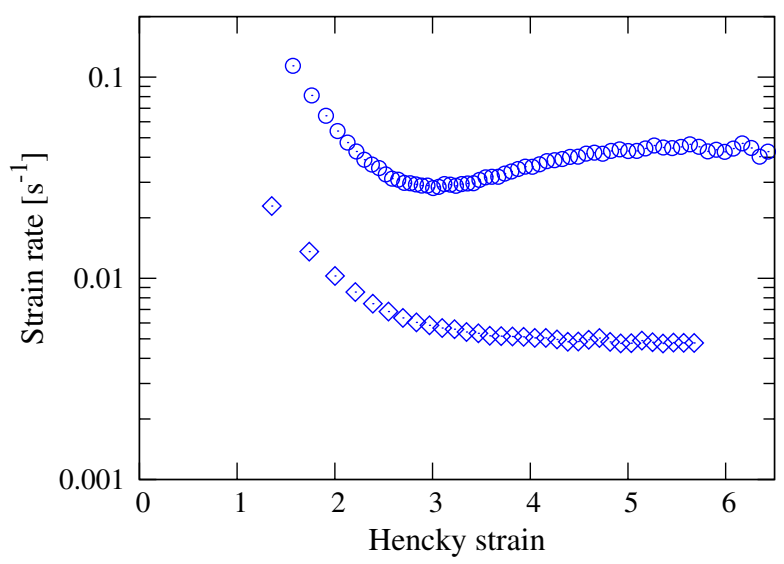

FIG. 4 (color online). Hencky strain rate $\dot{\epsilon}$ as a function of Hencky strain $\epsilon$ for constant applied $\sigma_{0}$ equal to $40 \mathrm{kPa}$ (diamonds, bottom) and $150 \mathrm{kPa}$ (circles, top). For $\sigma_{0}$ larger than approximately $80 \mathrm{kPa}$, the strain rate goes through a minimum before reaching a steady state value. 


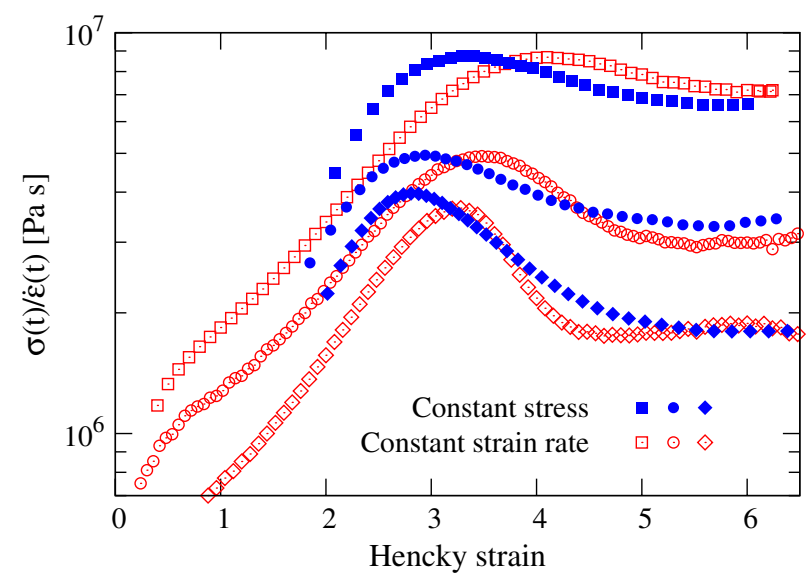

FIG. 5 (color online). $\sigma / \dot{\epsilon}$ as a function of Hencky strain $\epsilon$ for constant stress (closed symbols) and constant strain rate (open symbols) experiments. The constant stress experiments correspond to (filled squares) 80, (filled circles) 100, and (filled small diamonds) $200 \mathrm{kPa}$. The constant strain-rate experiments correspond to (squares) 0.01, (circles) 0.03, and (big diamonds) $0.1 \mathrm{~s}^{-1}$.

is only in the steady flow, creep is the more effective path to reach that state [11].

The extensional compliance $\bar{J}(t)=\epsilon(t) / \sigma_{0}$ by definition is a factor of 3 smaller than the shear compliance $J(t)$.

Creep measurements. - The extensional compliance $\bar{J}(t)$ measured in the FSR is compared with the LVE prediction from Eq. (3) in Fig. 3. Departure from the full line is a manifestation of the nonlinear mechanical properties of the melt. A good agreement with LVE is obtained for an imposed stress $\sigma_{0}$ up to $1000 \mathrm{~Pa}$, but for larger imposed stresses, the melt is less compliant than that predicted by LVE. This is indeed to be expected as it corresponds to extensional strain hardening observed in controlled deformation experiments [4]. In Fig. 3 (bottom), we compare the extensional compliance measured in the FSR with the shear compliance measured in an ARES-G2. Also in shear, we observe a deviation from the LVE at stresses above $1000 \mathrm{~Pa}$, but the material becomes more compliant than predicted from LVE. This behavior corresponds to the shear thinning typically encountered in the start-up of steady shear flow. The deviation at times less than $10 \mathrm{~s}$ may be due to limitations in the dynamic control of the shear rheometer.

In creep, the ultimate steady extensional flow is characterized by a constant slope of the compliance or equivalently a constant value of $\dot{\epsilon}$ such as in the case for $\sigma_{0}=40 \mathrm{kPa}$ in Fig. 4. At stresses above approximately $80 \mathrm{kPa}$, we observe an inflection point in the compliance corresponding to a minimum in the slope before a steady flow is established. The phenomenon is illustrated in Fig. 4 for $\sigma_{0}=150 \mathrm{kPa}$ with a minimum occurring at approximately $\epsilon=3$. The minimum in Fig. 4 corresponds closely to the maximum in stress observed in controlled deformation experiments previously reported in Ref. [4]. This is

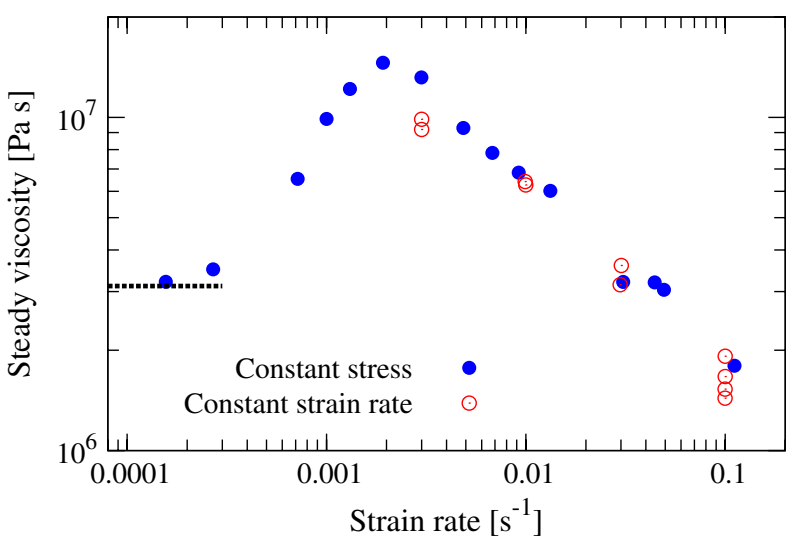

FIG. 6 (color online). Steady viscosity as a function of Hencky strain rate $\dot{\epsilon}$ for constant stress (closed symbols) and constant strain-rate (open symbols) experiments [4]. The dashed line represents the zero shear rate viscosity determined from the LVE.

illustrated further in Fig. 5, where the ratio of $\sigma / \dot{\epsilon}$ is plotted as a function of Hencky strain for the two extensional rheometry protocols: constant strain rate $[\dot{\epsilon}$ constant and $\sigma=\sigma(t)]$ and constant stress $\left[\sigma=\sigma_{0}\right.$ and $\left.\dot{\boldsymbol{\epsilon}}=\dot{\boldsymbol{\epsilon}}(t)\right]$. While there is no a priori reason to expect the two ratios to be the same, the similarity between the paths could indicate that the molecular mechanism behind the stress maximum is also behind the inflection point in the compliance [23].

Another important observation from Fig. 5 is that both protocols eventually approach constant values of $\sigma / \dot{\epsilon}$ lasting approximately 1-2 Hencky strain units before the measurements are terminated due to insufficient resolution. The steady flow state after the maximum can only be reached with an active control on the FSR. The steady ratios are identified as the extensional viscosity at the given stretch rates [23].

In Fig. 6, we compare our measurements of steady extensional viscosity obtained in creep with values obtained in controlled deformation [4]. Within experimental accuracy the two protocols give the same extensional viscosity. The extensional viscosity exhibits a maximum and an ultimate power-law behavior with the viscosity scaling approximately as $\dot{\boldsymbol{\epsilon}}^{-0.5}$ over almost two decades in $\dot{\boldsymbol{\epsilon}}$.

Conclusions.-The experimental findings support the existence of a stress maximum in fast stretching of branched polymer melts. The stress scaling at steady state after the maximum suggests that the maximum marks a transition to a flow state in which branched polymers behave as linear polymers. Indeed, the current scenario for relaxation in branched melts in the LVE regime [1-3] considers a hierarchy of relaxation (from outside to inside) and an effective conversion of a branched chain into a linear chain. If we assume the same conversion in the nonlinear regime, the $\dot{\epsilon}^{-0.5}$ decrease of the viscosity corresponds to the same power-law behavior found for linear melts [24].

The work was supported by The Danish Council for Independent Research-Technology and Production 
Sciences (10-082409), Højteknologifonden (LANI, 0112011-3), and the European Union (ITN, 214627, Dynacop). We would like to thank Professor R. G. Larson for useful comments.

Appendix.-Initial conditions for Eq. (1) become

$$
\sigma_{i}(0)=\frac{g_{i}}{G_{0}} \sigma_{0},
$$

where $G_{0}=\sum_{i} g_{i}$. Equations (1), (2), and (A1) are conveniently solved by Laplace transformation. The solution for $Y(s)=\int_{0}^{\infty} \dot{\gamma}(t) \exp (-s t) d t$ becomes

$$
Y(s)=\frac{\sigma_{0}}{G_{0}} \frac{\sum_{i}\left[g_{i} /\left(1+s \tau_{i}\right)\right]}{s \sum_{i}\left[g_{i} \tau_{i} /\left(1+s \tau_{i}\right)\right]} .
$$

The inverse transformation is performed by standard methods to yield the expression in Eq. (3). The retardation times are given as $\lambda_{k}=-1 / s_{k}$, where the $s_{k}$ are the zeros of

$$
S(s)=\sum_{i} \frac{g_{i} \tau_{i}}{1+s \tau_{i}} .
$$

The compliance coefficients are given after some simplification as

$$
j_{k}=\frac{\lambda_{k}^{2}}{\sum_{i}\left[g_{i} \tau_{i}^{2} /\left(1+s_{k} \tau_{i}\right)^{2}\right]} .
$$

The same result was obtained by Sips [13] but by a different procedure.

*oh@kt.dtu.dk

[1] D. Read, D. Auhl, C. Das, J. Doelder, M. Kapnistos, I. Vittorias, and T. McLeish, Science 333, 1871 (2011).

[2] L. Hutchings, S. Kimani, D. Hoyle, D. Read, C. Das, T. McLeish, T. Chang, H. Lee, and D. Auhl, ACS Macro Lett. 1, 404 (2012).

[3] R. G. Larson, Science 333, 1834 (2011).
[4] H. K. Rasmussen, J. K. Nielsen, A. Bach, and O. Hassager, J. Rheol. 49, 369 (2005).

[5] T. Burghelea, Z. Stary, and H. Munstedt, J. NonNewtonian Fluid Mech. 166, 1198 (2011).

[6] H. K. Rasmussen and O. Hassager, J. Non-Newtonian Fluid Mech. 171, 106 (2012).

[7] T. Burghelea, Z. Stary, and H. Munstedt, J. NonNewtonian Fluid Mech. 171, 107 (2012).

[8] Y. Wang, P. Boukany, S. Q. Wang, and X. Wang, Phys. Rev. Lett. 99, 237801 (2007).

[9] A. Lyhne, H. K. Rasmussen, and O. Hassager, Phys. Rev. Lett. 102, 138301 (2009).

[10] T. C. B. McLeish and R. Larson, J. Rheol. 42, 81 (1998).

[11] H. Münstedt, S. Kurzbeck, and L. Egersdörfer, Rheol. Acta 37, 21 (1998)

[12] R. J. Andrade and J. M. Maia, J. Rheol. 55, 925 (2011).

[13] R. Sips, J. Polym. Sci. 5, 69 (1950).

[14] Sips [13] was able to use the LVE result to predict the small strain creep response of a poly(isobutylene) sample.

[15] G. H. McKinley and T. Sridhar, Annu. Rev. Fluid Mech. 34, 375 (2002).

[16] A. Bach, H. Rasmussen, and O. Hassager, J. Rheol. 47, 429 (2003).

[17] J. Meissner, J. Rheol. 16, 405 (1972).

[18] M. Stadlbauer, H. Janeschitz-Kriegl, M. Lipp, G. Eder, and R. Forstner, J. Rheol. 48, 611 (2004).

[19] E. Nordmeier, U. Lanver, and M.D. Lechner, Macromolecules 23, 1072 (1990).

[20] E. Nordmeier, U. Lanver, and M. Lechner, Macromolecules 23, 1077 (1990).

[21] R. Bird, R. C. Armstrong, and O. Hassager, Dynamics of Polymeric Liquids (Wiley, New York, 1987), Vol. I.

[22] M. Wagner and V. Rolon-Garrido, J. Rheol. 56, 1279 (2012).

[23] Note that the steady viscosity is not dependent on the rate at which the control scheme reaches the setpoint; see Fig. S1. Additionally, it should be noted that the top plate trajectories undergo two very different paths; see Fig. S.2. See Supplemental Material at http://link.aps.org/ supplemental/10.1103/PhysRevLett.110.168301.

[24] A. Bach, K. Almdal, H. Rasmussen, and O. Hassager, Macromolecules 36, 5174 (2003). 